\title{
Bony orbital development after early enucleation in humans
}

\author{
Christoph Hintschich, Frans Zonneveld, Lelio Baldeschi, Catey Bunce, Leo Koornneef
}

\begin{abstract}
Aim-To analyse the extent of bony orbital volume reduction after enucleation in humans.

Methods-Volumetric studies on bony orbital volumes based on three dimensional reconstructions acquired from high resolution computed tomograph (CT) scans were performed in 29 patients with acquired anophthalmia and four patients before enucleation (controls). Eight patients (follow up 25-52 years) were enucleated in childhood aged between 0.4 and 8 years (group I), 21 in adulthood aged between 15 and 53 years. Fifteen of these patients (group IIa) had long standing anophthalmia (follow up 7-53 years), six patients (group IIb) were enucleated 9
\end{abstract} months to 4 years before CT.

Results-Bony orbital volumes were reduced in all patients with long standing anophthalmia. The median percentage reduction in enucleated orbits was $7.0 \%$ in group I, $3.8 \%$ in group IIa, and $1.9 \%$ in group IIb. In patients with long standing anophthalmia (I and IIa) the reductions were statistically significantly different ( $p$ $<0.01$ ) from zero. There was some evidence of a correlation between orbital volume reduction and age at enucleation (rho $=0.36, p=0.09$, Spearman rank correlation coefficient) and a statistically significant correlation between volume reduction and time interval since enucleation (rho $=-0.5, p=0.003)$. Clinically none of the patients showed significant facial asymmetry.

Conclusions-These data provide strong evidence that enucleation both in children and adults is associated with a reduction of bony orbital volume and that this decrease in volume is associated with increasing time. However, the reduction is smaller than generally assumed and does not cause obvious facial asymmetry. It is more related to the time interval since enucleation than the age at enucleation, which makes a mechanism of volume adaptation more likely than just retardation of growth.

(Br F Ophthalmol 2001;85:205-208)

Correspondence to:

Dr C Hintschich,

Department of

Ophthalmology,

Ludwig-Maximilians-

Universität, Mathildenstrasse

8, D-80336 München,

Germany

c.hintsch@ak-i.med.

uni-muenchen.de

Accepted for publication 15 June 2000

Orbital growth is believed to be checked following enucleation in childhood, resulting in malformation and facial asymmetry depending upon upon the age at which enucleation was performed. ${ }^{12}$ This was already suspected in the 19 th century, when the first experiments on animals were performed. ${ }^{3}$ Based on animal

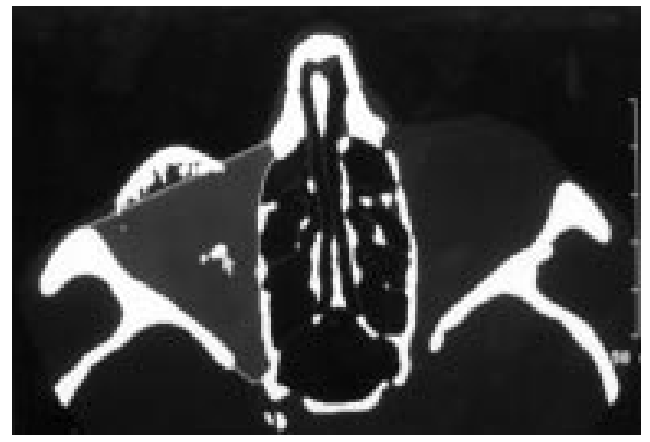

Figure 1 Segmentation of the right orbit. Grey areas compare with identical thresholds (min - $204 \mathrm{H} /$ max $+166 \mathrm{H}$ ). Stippled area outlined corresponds to the bony orbital volume. White dots (arrow) belong to the orbital implant.

research, Kennedy ${ }^{5}$ and Sarnat ${ }^{6-8}$ supported and finally established the theory of the detrimental influence of early enucleation on orbital growth. This belief, however, rarely corresponds to our clinical observations in humans and the appearance of anophthalmic patients enucleated in childhood. The interesting question as to what extent bony orbital growth is affected by early enucleation in humans remains unanswered. We attempt to address this question.

\section{Patients and methods}

Twenty nine patients ( 14 female, 15 male) with acquired anophthalmia were examined clinically and with high resolution computed tomography (CT). Patients with conditions possibly causing facial asymmetry, such as orbital fractures, phthisical eye, congenital or

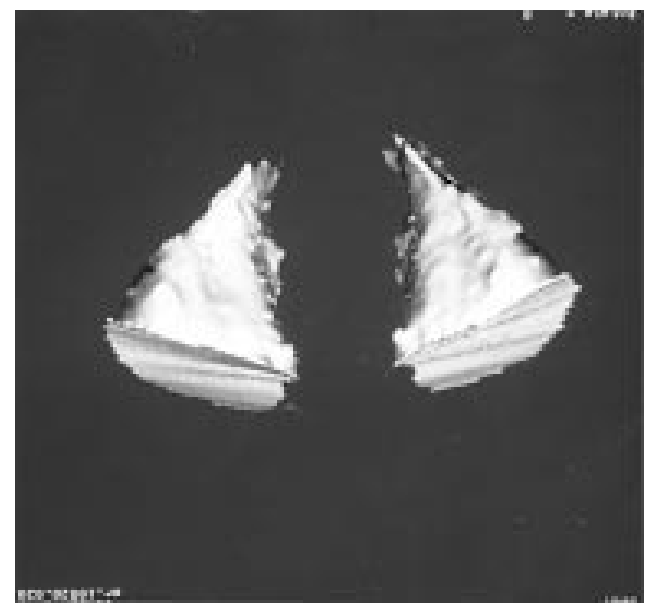

Figure 2 Bony orbital volume compartment,

corresponding to an impression mould of the orbit. Thirty five year old patient, enucleated with primary orbital implant age 3.5 years. $B O V_{\text {enuc }}$ (right): $25.5 \mathrm{ml}$, BOV: 27.1 $\mathrm{ml}, \mathrm{BOV}$ difference between enucleated and fellow orbit $-5.5 \%$. 


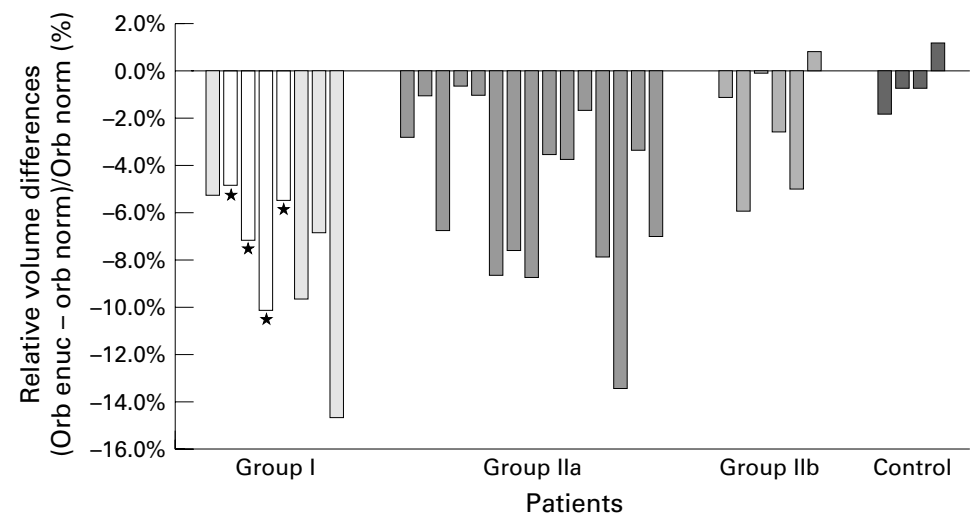

Figure 3 Bony orbital volume following enucleation. Relative volume differences, single values. Group I: childhood, long standing, group IIa: adulthood, long standing, group IIb: adulthood, short follow up. Negative values indicating smaller volume in the anophthalmic orbit. ${ }^{\star}$ Primary orbital implant.

acquired microphthalmia, and buphthalmos before enucleation, were not included. Eight patients with long standing anophthalmia of 25-52 years had been enucleated in childhood aged between 0.4 and 8 years (group I); one half of them received a primary orbital implant (18 $\mathrm{mm}$ Allen implant). The remaining 21 patients were enucleated in adulthood aged between 15 and 53 years. Fifteen of these patients (group IIa) also had long standing anophthalmia of 7-53 years, whereas six patients (group IIb) had short follow ups of 9 months to 4 years after enucleation. Four patients before enucleation were used for controls.

A CT scanning technique with $1.5 \mathrm{~mm}$ contiguous slices in a transverse plane was applied with the patients in a supine position (Philips Tomoscan SR 7000, Eindhoven, Netherlands). Three dimensional reconstruction was performed based on interactive segmentation of the cross sectional CT images at a Gyroview HP workstation (Philips Medical Systems, Eindhoven, Netherlands) with Gyroview software, version 5.0.3 (ISG Technologies Inc, Mississauga, Canada). The lower threshold for orbital volume segmentation was $-204 \mathrm{H}$, the upper $+166 \mathrm{H}$. High density structures such as orbital implants or artificial eyes were segmented separately, using thresholds of $+166 \mathrm{H}$ and $+2911 \mathrm{H}$ (maximum). For low density structures such as free air behind an artificial eye the thresholds were $-204 \mathrm{H}$ and $-1024 \mathrm{H}$ (minimum) respectively. The margins of the bony orbit were defined by the orbital walls and the orbital entrance, which was formed by the superior, lateral and inferior orbital rim, the anterior lacrimal crest, and a line connecting the anterior lacrimal crest with the superior orbital rim. ${ }^{9}$ The optic canal, the nasolacrimal duct, and the pterygopalatine fossa were not included (Fig 1). The volume confined by these areas, completed by the volume of implants, artificial eyes, or air, if present, is defined as the "bony orbital volume" (BOV). The volumes were calculated by voxel counting. Analysis and evaluation in this study are based on intraindividual differences between BOV of the anophthalmic and the fellow normal orbit. Additionally the patients were examined for clinical signs of facial asymmetry and photodocumented.

For quantitative analysis relative volume differences between anophthalmic and normal orbit were calculated:

$\left(\mathrm{BOV}_{\text {enuc }}-\mathrm{BOV}\right) / \mathrm{BOV}$

Non-parametric methods were used for analysis because of smallish numbers involved. The sign test was conducted to assess whether the average difference between the anophthalmic and normal orbit was statistically significantly different from zero. The Mann-Whitney rank sum test was used to assess whether reductions in different groups of patients were statistically significantly different. To assess association between orbital volume and each age at enucleation and time since enucleation, the Spearman rank correlation coefficient was calculated.

\section{Results}

Three dimensional reconstructions and volumetric data were obtained from all 33 patients. The image of the three dimensional reconstructed orbit corresponds to an impression mould of the orbit (Fig 2). Repeated segmentations and volume calculations of the same orbit revealed a variation of plus or minus $0.9 \%$, indicating the high precision of the method.

These data provide strong evidence ( $p$ $<0.01$ ) of lower anophthalmic orbit volume than normal orbit volume in all patients with long standing anophthalmia, both in patients enucleated in childhood and in adulthood. In group I there was a median change of $-1.7 \mathrm{ml}$ (interquartile range $-3.0,-1.5 \mathrm{ml}$ ), corresponding a relative volume reduction of $7.0 \%$ $(5.4 \%, 9.94 \%)$ of the enucleated orbit. In group IIa there was an absolute median BOV difference between the enucleated orbit and the normal orbit of $-0.9 \mathrm{ml}(-2.13,-0.41 \mathrm{ml})$, which is a $3.8 \%$ relative difference $(-7.90 \%$, $-1.75 \%)$. In both groups the differences were statistically significantly different from zero, the differences between groups were not significant.

Table 1 Absolute and relative bony orbital volumes (BOV) after enucleation

\begin{tabular}{|c|c|c|c|c|c|}
\hline & & $\begin{array}{l}\text { Age at enucleation } \\
\text { (years) }\end{array}$ & $\begin{array}{l}\text { Time between } \\
\text { enucleation and } C T \\
\text { (years) }\end{array}$ & $\begin{array}{l}\text { BOV difference } \\
\text { enucleation - norm }(\mathrm{ml})\end{array}$ & Relative BOV difference \\
\hline Patients & No & Median (range) & Median (range) & Median (IQ range) & Median (IQ range) \\
\hline I & 8 & $3.3(0.4-8)$ & $30.5(25-52)$ & $-1.7(-3.0,-1.5)$ & $-7.0 \%(-9.9 \%,-5.4 \%)^{\star}$ \\
\hline II a & 15 & $23(15-52)$ & $12(7-53)$ & $-0.9(-2.1,-0.4)$ & $-3.8 \%(-7.9 \%,-1.7 \%)^{\star}$ \\
\hline II b & 6 & $41(25-53)$ & $1.4(0.8-4)$ & $-0.4(-1.4,-0.01)$ & $-1.9 \%(-5.0 \%, 0 \%)$ \\
\hline Controls & 4 & & & $-0.19(-0.38,0.05)$ & $-0.8 \%(-1.3 \%, 0.15 \%)$ \\
\hline
\end{tabular}

${ }^{\star} \mathrm{p}<0.01, \mathrm{IQ}=$ interquartile. 


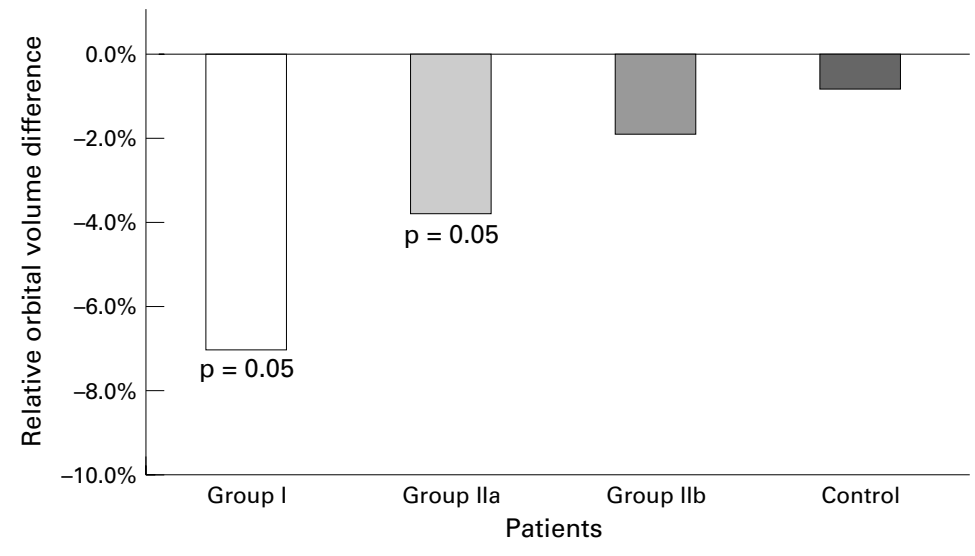

Figure 4 Bony orbital volume following enucleation. Relative volume differences, median.

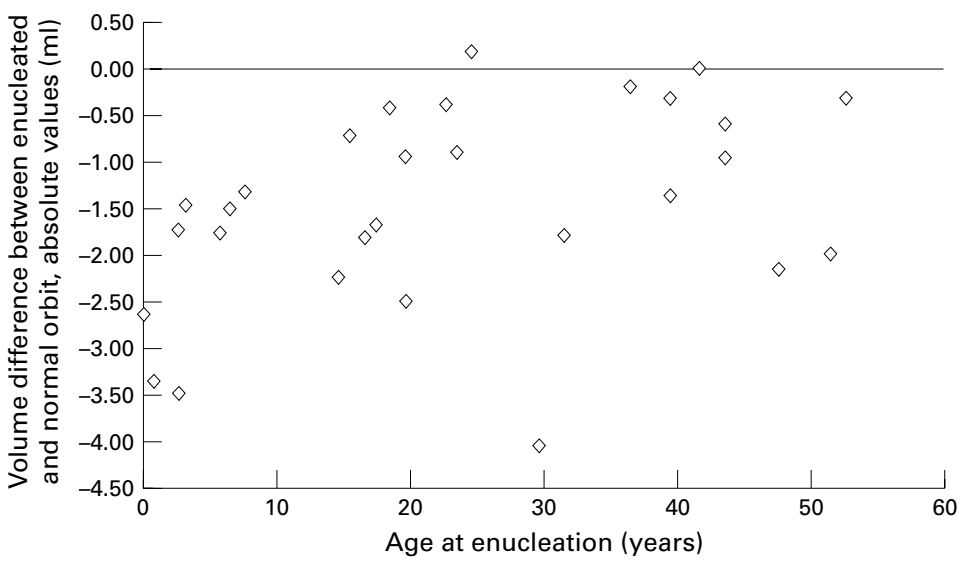

Figure 5 Scatter diagram. Age at enucleation versus BOV difference enucleated orbit normal orbit. rho $=0.36, p=0.09$, Spearman rank order correlation.

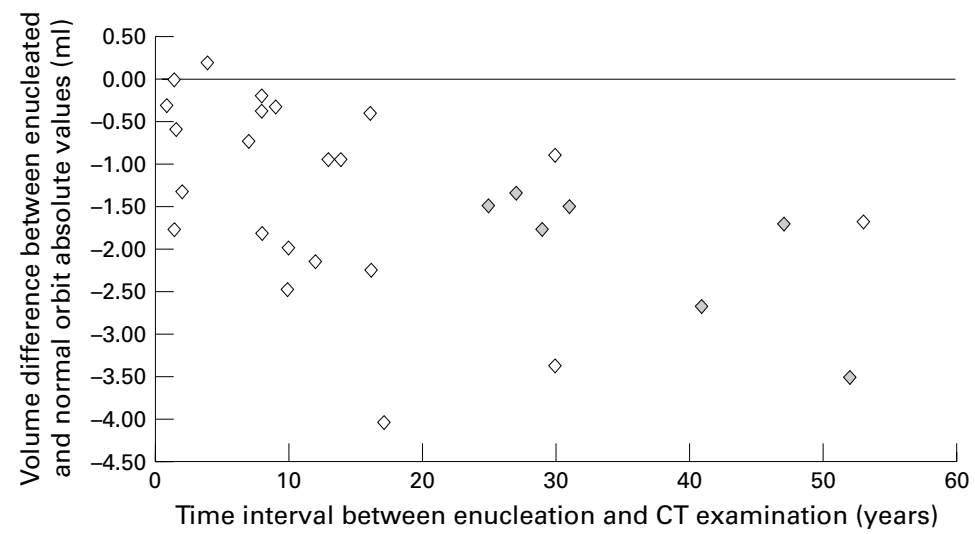

Figure 6 Scatter diagram. Time interval enucleation to CT examination versus BOV difference enucleated orbit - normal orbit. rho $=-0.56, p=0.003$, Spearman rank order correlation. Grey diamond = patients enucleated in childhood.

There was also a mild, but insignificant, decrease of the BOV in group IIb of $-0.4 \mathrm{ml}$ $(-1.3,-0.01 \mathrm{ml})$, corresponding to $-1.9 \%$ relative difference $(-5.03 \%, 0 \%)$. The greatest reduction seen in the four controls was $-1.8 \mathrm{ml}$ (Table 1).

The relative orbital volume differences for each patient and controls are shown in Figure 3. There were no differences in BOV reduction between patients with and without primary orbital implants.

Median values of all groups are illustrated in Figure 4.

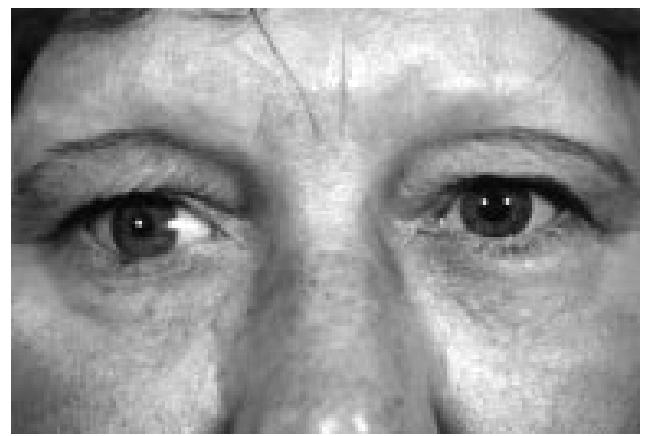

Figure 7 Fifty five year old patient enucleated at age 3 years, after secondary orbital implant. $B O V_{\text {enuc }}: 20.2 \mathrm{ml}$, BOV: 23.7 ml, BOV difference: $-14.7 \%$. No clinically obvious orbital malformation or facial asymmetry.

The relation between age at enucleation and BOV is shown in a scatter diagram (Fig 5) for patients in groups I and IIa. We found some evidence (not statistically significant) of a positive correlation between the age at enucleation and the BOV difference (rho $=0.36, \mathrm{p}=0.09$ ). There was evidence, however, of a correlation between the influence of the time interval since enucleation and the BOV difference (rho = $-0.56, p=0.003)($ Fig 6).

Clinically none of the patients showed signs of facial asymmetry. This is even true for the patient with the largest relative volume difference of $-14.7 \%$ (Fig 7 ).

\section{Discussion}

"Among the apparent truisms in ophthalmology, there is one, at least, which requires close scrutiny. This is the belief that orbital growth is checked following enucleation in childhood, resulting in more or less maldevelopment and facial asymmetry depending upon the age at which enucleation was performed." With these sentences Howard begins his article on orbital growth after unilateral enucleation in childhood. ${ }^{10}$ However, in a series of 15 patients enucleated under the age of 12 years he did not find a significant decrease of the orbital size or an asymmetry. He concluded that enucleation of one eye in infancy or childhood is associated with a small reduction in the size of the bony orbit in later years, but that this reduction is not of cosmetic consequence. The same opinion had been expressed by Gordon Byers from Moorfields Eye Hospital in 1898, based on his study on 10 patients enucleated during childhood. ${ }^{11}$ Following his report he detected no arrest of development. His observations were questioned by others. ${ }^{12}{ }^{13}$ Thomson ${ }^{12}$ carried out experimental enucleations in young rabbits. His results have been considered to show conclusively that, in rabbits, a deficiency of growth occurs in the anophthalmic orbit. In the 1960s Kennedy ${ }^{5}$ and Sarnat ${ }^{6}$ began their $^{2}$ extensive studies on the effect of early enucleation on the orbit. They found significant reductions of the orbital size particularly in the animal model. Based on approximate estimations, Kennedy ${ }^{5}$ calculated the decrease in the orbital volume up to $19.5 \%$ in patients with orbital implants and up to $27.7 \%$ in patients without orbital implants. He assumed that the volume reduction after enucleation can reach 
the magnitude of $35-50 \%$. Their work was of fundamental importance to the understanding of the problem of early enucleation and persistently influenced the point of view of ophthalmologists. Up to now it has been generally accepted that early enucleation causes harmful maldevelopment of the enucleated orbit. ${ }^{14-20}$

Can our results on three dimensional reconstructions and orbital volumetry in anophthalmic patients add any new aspects to this thoroughly discussed issue? Yes, because for the first time exact volumetric data on a larger series of anophthalmic humans in vivo with very long follow ups are available. Our volumetric studies based on high resolution CT scans confirm a reduction of the anophthalmic bony orbit in patients enucleated in their early childhood. However, the volume reduction is smaller than generally assumed. In our patients we found that the greatest reduction of the anophthalmic orbit compared with the fellow orbit was $-14.5 \%$ which is less than the few volumetric data given in the literature, where volume reductions were calculated at between $19.5 \%$ and $50 \% .^{521}$

Interestingly, we also found a statistically significant reduction of the BOV in patients who were enucleated when they were grown up. In this adult group the volume deficit reached up to $4 \mathrm{ml}$ and the median reduction almost $-4 \%$ in the enucleated orbit 12 years after enucleation. The adult bony orbit has been regarded as a stable compartment not being influenced by enucleation. ${ }^{182-25}$ This is obviously not the case. However, there are some early observations suggesting that enucleation might induce shrinkage of the bony orbit ${ }^{26}{ }^{27}$ which had not yet been proved.

Correlation analysis of $\mathrm{BOV}$ changes versus age at enucleation and time interval since enucleation shows a stronger association between reduction of BOV and the time course than age at surgery.

There was no difference between patients with and without primary orbital implants, suggesting that the post-enucleation bony orbital development in humans may not be influenced by implants. Small numbers (4/4) make statistical analysis not applicable. However, in none of our patients was clinically obvious facial asymmetry noticed. This is consistent with reports in the literature on patients who had been enucleated during childhood and who showed no significant facial malformation despite differences in radiological features. ${ }^{1022}$ Recently Fountain et al examined nine patients 5-10 years after enucleation during early childhood (age $0.4-6$ years). Morphometric measurements of five linear orbital dimensions using a millimetre rule were collected. They found no statistically significant difference in any of the measured orbital dimensions between the enucleated and fellow orbits, which was attributed to the presence of an orbital implant. ${ }^{28}$ However, their observa- tion, which is consistent with our clinical impression, is more understandable because of the small volume reductions, which cannot be detected by simple and rough linear measurements than the fact, that all nine patients had primary orbital implants. In our series at least there is no evidence that orbital implants influence BOV after early enucleation.

Supported by Deutsche Forschungsgemeinschaft (DFG), Bupported by Deutsche Forschungsgemeinsch

1 Duke-Elder WS. Textbook of ophthalmology. London: Henry Kimpton, 1932:363.

2 Whitnall SE. Anatomy of human orbit and accessory organs of vision. London: Humphrey Milford, 1932:98-108.

3 Fick. Über die Ursachen der Knochenformen. Göttingen, 1857. 4 Gudden v. Experimental-Untersuchungen über das Schädelwachstum. München, 1874.

5 Kennedy RE. The effect of early enucleation on the orbit in animals and humans. Trans Am Ophthalmol Soc 1964;62: 459.

6 Sarnat BG, Shanedling PD. Postnatal growth of the orbit and upper face in rabbits after exenteration of the orbits. Arch Ophthalmol 1965;73:829-37.

7 Sarnat BG. Orbital volume after enucleation and eye volume in the adult rabbit. Graefes Arch Ophthalmol 1978;208:241245.

8 Sarnat BG. Eye volumes in young and adult rabbits. Acta Anat 1980;109:462-7.

9 Zonneveld FW, Meulen van der JC, Koornneef L, et al. Three-dimensional CT imaging of the orbit. In: JC Meulen van der, JS Gruss, JC Meulen van der, JS Grusss. Color atlas and text of ocular plastic surgery. London: Mosby-Wolfe, 1996:9-30.

10 Howard GM, Kinder RSL, MacMillan AS. Orbital growth after unilateral enucleation in childhood. Arch Ophthalmol 1965;73:80-3

11 Byers W. Report of the Commitee of the Ophthalmic Society on excision. Trans Ophthalmol Soc UK 1898;18:256-7.

12 Thomson WE. The determination of the influence of the eyeball on the growth of the orbit by experimental enucleaeyeball on the growth of the orbit by experimental enuclea-
tion of one eye in young animals. Trans Ophthalmol Soc UK 1901;21:258-263.

13 Wessely K. Beiträge zu den Wachstumsbeziehungen wischen dem Augapfel und seinen Nachbarorganen. Graefes Arch Ophthalmol 1921;105:491-501.

14 Apt L, Isenberg S. Changes in orbital dimensions following enucleation. Arch Ophthalmol 1973;90:393-5.

5 Van Limborgh J, Tonneyck-Müller I. Orbital growth pattern in experimental microphthalmia. Modern Problems in Ophthalmology 1975;14:1-4

16 Amemiya T, Matsumara M, Hirose Y. Effects of radiation after enucleation without implantation on orbital developement of patients with retinoblastoma. Ophthalmologica 1977; 174:137-44.

17 Cepela MA, Nunery WR, Martin RT. Stimulation of orbital growth by the use of expandable implants in the anophthalmic cat orbit. Ophthalmic Plast Reconstr Surg 1992;8:157-69.

18 Kennedy RE. The effect of early enucleation on the orbit in animals and humans. Adv Ophthalmic Plast Reconstr Surg 1992;9:1-39.

19 Eppley BL, Holley S, Sadove AM. Experimental effects of intraorbital tissue expansion on orbitomaxillary growth in anophthalmos. Ann Plast Surg 1993;31:19-26.

20 Heinz GW, Nunery WR, Cepela MA. The effect of maturation on the ability to stimulate orbital growth using tissue expanders in the anophthalmic cat orbit. Ophthal Plast Reconstr Surg 1997;13:115-28.

21 Kennedy RE. Bone changes in the adult anophthalmic orbit influencing oculoplastic reconstructive considerations. Trans Am Ophthalmol Soc 1976;74:237-48.

22 Honegger H, Müller-Staufenbiel H. Das Wachstum der Orbita nach Enukleation im frühen Kindesalter und ihr Einflu $\beta$ auf das kosmetische Spätergebnis. Klin Monatsbl Augenheilkd 1967;150:655-63.

23 Luzsa E. Der Einfluß der Enukleation auf die Entwicklung des Sehnervenkanals. Klin Monatsb Augenheilkd 1938;101: des Sehn.

24 Taylor WOG. The effect of enucleation of one eye in childhood upon the subsequent developement of the face. Trans Ophthalmol Soc UK 1939;59:361-9.

25 Osborne D, Hadden OB, Deeming LW. Orbital growth after childhood enucleation. Am f Ophthalmol 1974;77:756-9.

26 Weiss L. Ueber das Verhalten der Augenhöhle bei Einäugigen. Arch Augenheilk 1892;25:423-46.

27 Pfeiffer RL. The effect of enucleation on the orbit. Trans Am Acad Ophth Otolaryngol 1945;49:236-7.

28 Fountain T, Goldberger S, Murphree A. Orbital developement after enucleation in early childhood. Ophthal Plast Reconstr Surg 1999;15:32-6. 